\title{
Anomalous spin waves and the commensurate-incommensurate magnetic phase transition in LiNiPO4
}

Jensen, Thomas Bagger Stibius; Christensen, Niels Bech; Kenzelmann, M.; Ronnow, H.M.; Niedermayer, C.; Andersen, Niels Hessel; Lefmann, Kim; Jimenez-Ruiz, M.; Demmel, F.; Li, J.

Total number of authors:

12

Published in:

Physical Review B Condensed Matter

Link to article, DOI:

10.1103/PhysRevB.79.092413

Publication date:

2009

Document Version

Publisher's PDF, also known as Version of record

Link back to DTU Orbit

Citation $(A P A)$ :

Jensen, T. B. S., Christensen, N. B., Kenzelmann, M., Ronnow, H. M., Niedermayer, C., Andersen, N. H., Lefmann, K., Jimenez-Ruiz, M., Demmel, F., Li, J., Zarestky, J. L., \& Vaknin, D. (2009). Anomalous spin waves and the commensurate-incommensurate magnetic phase transition in LiNiPO4. Physical Review $B$ Condensed Matter, 79(9), 092413. https://doi.org/10.1103/PhysRevB.79.092413

\section{General rights}

Copyright and moral rights for the publications made accessible in the public portal are retained by the authors and/or other copyright owners and it is a condition of accessing publications that users recognise and abide by the legal requirements associated with these rights.

- Users may download and print one copy of any publication from the public portal for the purpose of private study or research.

- You may not further distribute the material or use it for any profit-making activity or commercial gain

- You may freely distribute the URL identifying the publication in the public portal 


\title{
Anomalous spin waves and the commensurate-incommensurate magnetic phase transition in $\mathrm{LiNiPO}_{4}$
}

\author{
T. B. S. Jensen, ${ }^{1}$ N. B. Christensen, ${ }^{1,2,3}$ M. Kenzelmann, ${ }^{2,4}$ H. M. Rønnow, ${ }^{2,5}$ C. Niedermayer, ${ }^{2}$ N. H. Andersen, ${ }^{1}$ \\ K. Lefmann, ${ }^{1,3}$ M. Jiménez-Ruiz, ${ }^{6}$ F. Demmel, ${ }^{7}$ J. Li, ${ }^{8}$ J. L. Zarestky, ${ }^{8}$ and D. Vaknin ${ }^{8}$ \\ ${ }^{1}$ Materials Research Division, Risф DTU, National Laboratory for Sustainable Energy, Technical University of Denmark, \\ DK-4000 Roskilde, Denmark \\ ${ }^{2}$ Laboratory for Neutron Scattering, Paul Scherrer Institut and ETH Zürich, CH-5232 Villigen, Switzerland \\ ${ }^{3}$ Nano Science Center, Niels Bohr Institute, University of Copenhagen, Universitetsparken 5, 2100 København $\emptyset$, Denmark \\ ${ }^{4}$ Laboratory for Solid State Physics, ETH Zürich, CH-8093 Zürich, Switzerland \\ ${ }^{5}$ Laboratory for Quantum Magnetism, École Polytechnique Fédérale de Lausanne (EPFL), 1015 Lausanne, Switzerland \\ ${ }^{6}$ Institut Laue Langevin, BP 156, F-38042 Grenoble Cedex 9, France \\ ${ }^{7}$ Rutherford Appleton Laboratory, ISIS Facility, Didcot OX11 0QX, Oxon, England \\ ${ }^{8}$ Department of Physics and Astronomy and Ames Laboratory, Iowa State University, Ames, Iowa 50011, USA
}

(Received 30 January 2009; published 20 March 2009)

\begin{abstract}
Detailed spin-wave spectra of magnetoelectric $\mathrm{LiNiPO}_{4}$ have been measured by neutron scattering at low temperatures in the commensurate (C) antiferromagnetic (AF) phase below $T_{N}=20.8 \mathrm{~K}$. An anomalous shallow minimum is observed at the modulation vector of the incommensurate (IC) AF phase appearing above $T_{N}$. A linear spin-wave model based on Heisenberg exchange couplings and single-ion anisotropies accounts for all the observed spin-wave dispersions and intensities. Along the $b$ axis an unusually strong next-nearestneighbor AF coupling competes with the dominant nearest-neighbor AF exchange interaction and causes the IC structure.
\end{abstract}

DOI: 10.1103/PhysRevB.79.092413

PACS number(s): 75.25.+z, 75.10.Jm, 75.30.Ds, 75.50.Ee

Strongly correlated systems with electronic and magnetic interactions have attracted much attention recently. Noted among them are the high-temperature superconductors, the colossal-magneto-resistance materials, and the multiferroics. ${ }^{1}$ The latter group of materials possess magnetic and ferroelectric phases that may even coexist over a range of temperatures. ${ }^{2}$ For some, the correlations are manifested in the magnetoelectric (ME) effect in which a magnetic field induces an electric polarization and an electric field induces a magnetic moment. ${ }^{3}$ The lithium orthophosphates $\mathrm{LiMPO}$, with $M=\mathrm{Mn}, \mathrm{Fe}, \mathrm{Co}$, and $\mathrm{Ni}$, constitute a prototypical isostructural group of antiferromagnets $(\mathrm{AF})$ that are ME below their Néel temperatures. ${ }^{4,5}$ Although they have been investigated for long, ${ }^{4}$ a general consensus about the microscopic interactions leading to their ME effect has not yet been established. ${ }^{6,7}$

Here we focus on $\mathrm{LiNiPO}_{4}$ which compared to its lithium orthophosphate (LO) counterparts has more complex magnetic ordering properties and a different temperature dependence of the linear ME coefficients. ${ }^{4,8} \mathrm{LiNiPO}_{4}$ is orthorhombic with Pnma (no. 62) symmetry, lattice parameters $a=10.02 \AA, b=5.86 \AA, c=4.68 \AA$, and 4 f.u./unit cell. ${ }^{9}$ The magnetic $\mathrm{Ni}^{2+}$ ions with spin $S=1$ are located on the $4(c)$ sites and form buckled planes perpendicular to the crystallographic $a$ axis. All LO compounds have low-temperature zero-field commensurate (C) AF phases, but only $\mathrm{LiNiPO}_{4}$ passes via a first-order phase transition at $T_{N}=20.8 \mathrm{~K}$ into an incommensurate (IC) AF phase with ordering temperature $T_{\text {IC }}=21.7 \mathrm{~K}$. Above $T_{\text {IC }}$ short-range correlations persist up to $40 \mathrm{~K} .{ }^{8}$ In a recent study we have determined the magnetic structures and $(H, T)$ phase diagram as function of temperature and magnetic field up to $14.7 \mathrm{~T}$ applied along the $c$ axis. ${ }^{7}$ Here we also suggested a microscopic model account- ing for the symmetry and temperature dependence of the measured ME coefficients. ${ }^{8}$ The present inelastic neutron scattering study is aimed at determining the magnetic couplings in $\mathrm{LiNiPO}_{4}$ to improve our knowledge of the magnetic phases. Although the $\mathrm{C}$ phase has the general ordering vector $\mathbf{k}_{\mathrm{C}}=(0,0,0)$ we find an anomalous shallow minimum in the magnon dispersion along the $b$ direction at a modulation vector $\mathbf{q}_{m} \approx(0,0.12,0)$ (in reciprocal-lattice units). This is in the range of the IC ordering vector, $\mathbf{k}_{\mathrm{IC}}=(0, q, 0)$, where $q$ is increasing with temperature between $0.07<q<0.155 .{ }^{8}$ Our analyses of the spin-wave spectra show that the minimum at $\mathbf{q}_{m}$ results from competing nearest-neighbor (NN) and nextnearest-neighbor (NNN) AF interactions along the $b$ axis, and that the low-temperature zero field AF structure is on the verge between $\mathrm{C}$ and IC orders.

A high quality single crystal with approximate dimensions $5 \times 5 \times 9 \mathrm{~mm}^{3}$ and weight $0.4 \mathrm{~g}$ was used for this study. Inelastic neutron scattering measurements were performed on the RITA-II spectrometer (SINQ, PSI) by varying the incident energy at constant $q$ to obtain dispersions along $(q, 1,0)$ and $(0,1+q, 0)$. RITA-II uses a seven-blade analyzer and a final energy of $5 \mathrm{meV} .{ }^{10}$ Spin waves along the three principal directions, $(q, 1,0),(0,1+q, 0)$, and $(0,1, q)$ were measured on the HB1A spectrometer (HFIR, Oak Ridge) with $14.7 \mathrm{meV}$ initial energy and were within uncertainties the same as those measured on RITA-II. More than an entire Brillouin zone in the $b c$ plane was also measured on the IN8 spectrometer (ILL), using a 47 channel multianalyzer-detector (Ref. 11) set for a final energy of 30 $\mathrm{meV}$.

The spin-wave dispersions and integrated intensities measured at RITA-II and HB1A along the $a-, b-$, and $c$ directions at low temperatures are shown in Fig. 1 . The $(q, 1,0)$ and 

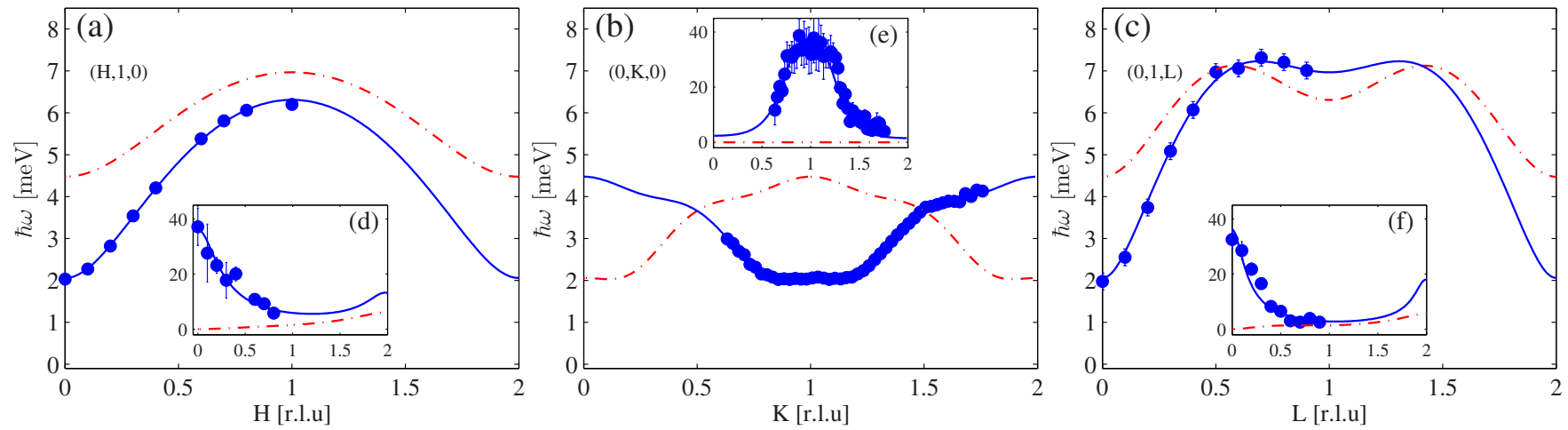

FIG. 1. (Color online) Measured (filled circles) spin-wave dispersion along three reciprocal directions, compared with a HolsteinPrimakoff spin-wave calculation (full and dotted lines) as explained in the text. The insets show the corresponding measured and calculated intensities (in arbitrary units) as function of wave vector. The dispersions along $(H, 1,0)$ and $(0, K, 0)$ were measured at $T=2 \mathrm{~K}$ on RITA-II, while the dispersion along $(0,1, L)$ was measured at $T=10 \mathrm{~K}$ on HB1A.

$(0,1, q)$ dispersions behave as expected for an antiferromagnet with an energy gap at the zone center, but the dispersion along $(0,1+q, 0)$ is unusual. It is almost a constant at low $q$ with a shallow minimum at $q \approx 0.12$, corresponding to the observed IC ordering vectors just below $T_{\mathrm{IC}} \cdot{ }^{8}$ Generally, the $(0,1+q, 0)$ modes are lower in energy than along the two other principal directions, and they soften the same way as the $q=0$ mode when the temperature increases toward the C-IC transition temperature $T_{N}$. Figure 2 shows the temperature dependence of the spin-wave gap at $\mathbf{Q}=(0,1,0)$. The inset displays spin-wave neutron scattering spectra at $\mathbf{Q}$ $=(0,1.055,0)$, close to the $q$ value of the IC-modulation vector found just above $T_{N}$. No spin-waves were observed in the IC phase.

As a basis for our spin-wave model we adopt the AF ground state of the $\mathrm{Ni}^{2+}$ ions determined by Santoro et al. ${ }^{12}$ and Vaknin et al. ${ }^{13}$ with antiparallel spins pointing along the $c$ axis as shown in Fig. 2(b). In fact, the spins are canted slightly away from the $c$ axis, ${ }^{7,14}$ consistent with a Dzyaloshinsky-Moria interaction, but this has negligible influence on the linear spin-wave model presented here. Ac-
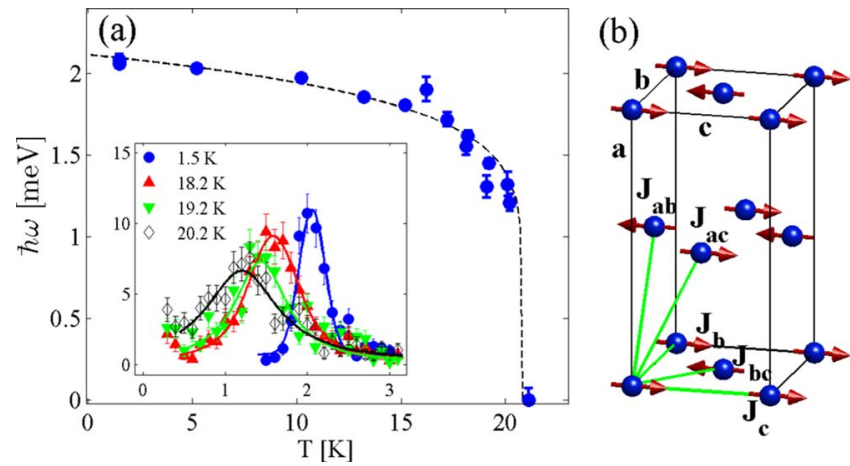

FIG. 2. (Color online) (a) Spin-wave energy gap at $\mathbf{Q}$ $=(0,1,0)$ as function of temperature measured at RITA-II. The dashed line is a guide to the eyes. The inset shows constant $\mathbf{Q}$ $=(0,1.055,0)$ scans at different temperatures with $\hbar \omega$ (in $\mathrm{meV}$ ) along the $x$ axis and measured intensity (in counts per minute) along $y$. (b) The magnetic unit cell of $\mathrm{LiNiPO}_{4}$ showing only $\mathrm{Ni}^{2+}$ ions. The depicted spin configuration and exchange interactions are the ones used in the spin-wave model. cordingly, we use the following spin Hamiltonian for $\mathrm{LiNiPO}_{4}$, with Heisenberg interactions $J_{i j}$ and standard single-ion anisotropy terms $D_{\alpha}\left(S^{\alpha}\right)^{2}(\alpha=a, b, c)$ :

$$
\mathcal{H}=\sum_{i, j} J_{i j} \mathbf{S}_{i} \cdot \mathbf{S}_{j}+\sum_{i, \alpha} D_{\alpha}\left(S_{i}^{\alpha}\right)^{2}
$$

In the $b c$ plane the $\mathrm{Ni}$ spins are coupled via superexchange $\mathrm{Ni}^{2+}-\mathrm{O}-\mathrm{Ni}^{2+}$ bonds, and our model includes $\mathrm{NN}$ interactions, $J_{b c}$, with a $3.81 \AA$ bond length, and two NNN, $J_{b}$ and $J_{c}$, with 4.71 and $5.89 \AA$ bonds, respectively. For the couplings between the $b c$ planes we consider NN interactions $J_{a b}$ and $J_{a c}$, which have 5.40 and $5.50 \AA$ bond lengths [see Fig. 2(b)]. The interplane couplings are mediated by $\mathrm{PO}_{4}$-tetrahedra and may have a significant magnitude, e.g., as found in $\mathrm{Li}_{3} \mathrm{Fe}_{2}\left(\mathrm{PO}_{4}\right)_{3}$. ${ }^{15}$

Using linear spin-wave theory we have calculated the spin-wave dispersions and intensities by a HolsteinPrimakoff approach similar to the one described in Ref. 16. The two spin-wave dispersions derived from Eq. (1) are given by (for details see Ref. 14),

$$
\hbar \omega=\sqrt{A^{2}-(B \pm C)^{2}},
$$

where

$$
\begin{gathered}
A \equiv 4 S\left(J_{b c}+J_{a b}\right)-2 S\left\{J_{b}\left[1-\cos \left(\mathbf{Q} \cdot \mathbf{r}_{5}\right)\right]\right. \\
+J_{c}\left[1-\cos \left(\mathbf{Q} \cdot \mathbf{r}_{6}\right)\right]+J_{a c}\left[2-\cos \left(\mathbf{Q} \cdot \mathbf{r}_{7}\right)\right. \\
\left.\left.-\cos \left(\mathbf{Q} \cdot \mathbf{r}_{8}\right)\right]\right\}+(S-1 / 2)\left(D_{a}+D_{b}\right), \\
B \equiv(S-1 / 2)\left(D_{a}-D_{b}\right),
\end{gathered}
$$

TABLE I. The fitted spin coupling constants for $\mathrm{LiNiPO}_{4}$. All units are in meV. By definition: $D_{c} \equiv 0 \mathrm{meV}$.

\begin{tabular}{ccccccc}
\hline \hline$J_{b c}$ & $J_{b}$ & $J_{c}$ & $J_{a b}$ & $J_{a c}$ & $D_{a}$ & $D_{b}$ \\
\hline $1.04(6)$ & $0.670(9)$ & $-0.05(6)$ & $0.30(6)$ & $-0.11(3)$ & $0.339(2)$ & $1.82(3)$
\end{tabular}



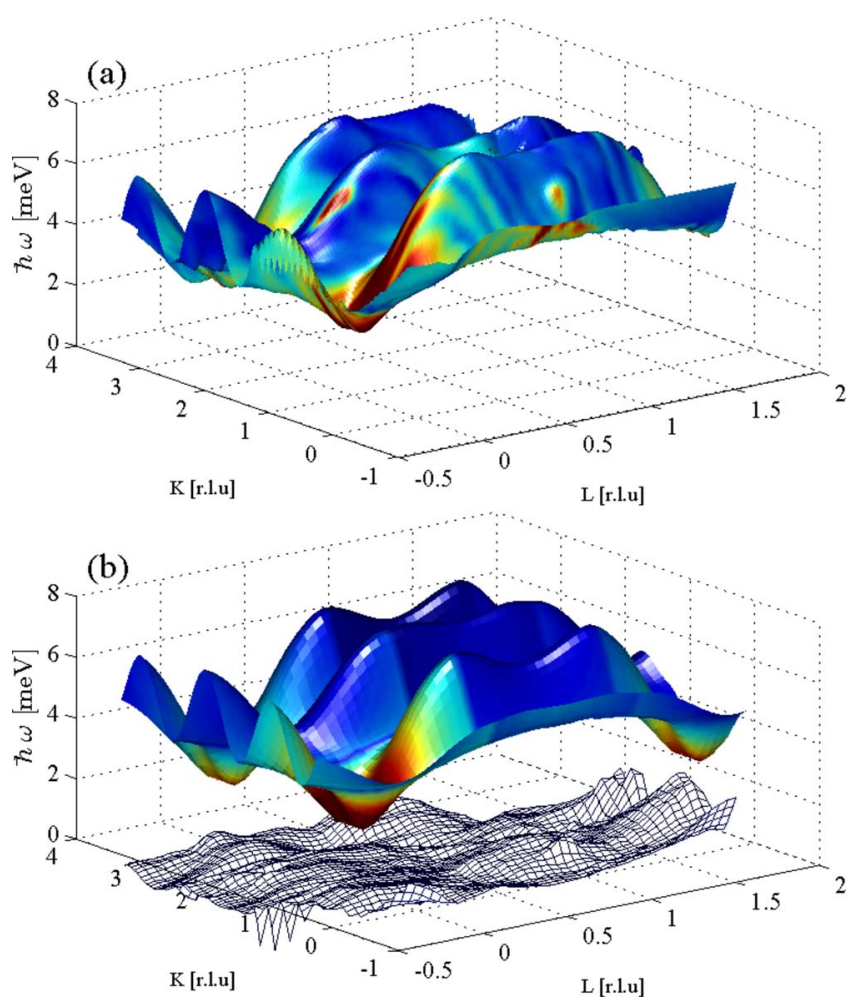

FIG. 3. (Color online) Spin-wave dispersions in the $(0, K, L)$ plane corresponding to the most intense branch. In colors we present spin-wave intensities with red for high and blue for low intensity. (a) Experimental data from IN8 at ILL. (b) Calculated data using the spin-wave model described in the text. The mesh around $0 \mathrm{meV}$ shows the deviation in energy between (a) and (b).

$$
\begin{aligned}
C \equiv & 2 J_{b c} S\left[\cos \left(\mathbf{Q} \cdot \mathbf{r}_{1}\right)+\cos \left(\mathbf{Q} \cdot \mathbf{r}_{2}\right)\right]+2 J_{a b} S\left[\cos \left(\mathbf{Q} \cdot \mathbf{r}_{3}\right)\right. \\
& \left.+\cos \left(\mathbf{Q} \cdot \mathbf{r}_{4}\right)\right] .
\end{aligned}
$$

Here $\mathbf{r}_{i}$ denotes vectors between $\mathrm{NN}$ or NNN Ni ions, and are: $\mathbf{r}_{1,2}=\frac{1}{2}(\mathbf{b} \pm \mathbf{c}), \quad \mathbf{r}_{3,4}=\frac{1}{2}(\mathbf{a} \pm \mathbf{b}), \quad \mathbf{r}_{5}=\mathbf{b}, \quad \mathbf{r}_{6}=\mathbf{c}$, and $\mathbf{r}_{7,8}$ $=\frac{1}{2}(\mathbf{a} \pm \mathbf{c})$.

The model parameters were determined by a simultaneous least-squares refinement of the spin-wave dispersions in all three principal directions. ${ }^{17}$ The best fit is shown by full and dotted lines in Fig. 1, and the refined parameters are listed in Table I. The couplings $J_{a c}$ and $J_{c}$ assure ferromagnetically ordered $a c$ planes that interact via $J_{b c}+J_{a b}$ to $\mathrm{NN}$ and $J_{b}$ to NNN $a c$ planes along the $b$ direction. All interactions $J_{b c}, J_{a b}$ $(\mathrm{NN})$, and $J_{b}(\mathrm{NNN})$ are AF which leads to frustration promoting the IC ordering. We note that the $J_{a b}$ and $J_{a c}$ couplings in the $a$ direction are somewhat weaker than $J_{b c}$ and $J_{b}$. This is consistent with the findings in Ref. 13 where magnetic short-range fluctuations extending into the paramagnetic regime and a critical exponent $\beta=0.12$ of the $\mathrm{C}$ order parameter indicates a near-two-dimensional magnetic ordering of the $b c$ planes. The single-ion anisotropies, $D_{a}$ and $D_{b}$, are both positive indicating that a $c$ axis magnetic moment is favored in the ground state, as observed experimentally. It should be noted that it is not possible from Eq. (2) to determine which of the two is larger, $D_{a}$ or $D_{b}$. However, comparing the calculated intensities to the measured ones (insets of Fig. 1) establishes unequivocally that $D_{a}$ $<D_{b}$, as given in Table I.

From the symmetry of the magnetic structure it can be shown that at least two nondegenerate dispersions are needed to account for the spin wave in the $b$ direction. ${ }^{14}$ In our model calculations the existence of two nondegenerate branches is a result of the different anisotropies along $a, b$, and $c$. However, at RITA-II and HB1A (Fig. 1) we observed only a single magnon dispersion, either because the second branch had too low intensity (see Fig. 1 insets), or because the two dispersions were indistinguishable within instrumental resolution at the measured $\mathbf{Q}$ values. Searching for the second branch and further confirmation of our model, we used the IN8 spectrometer to collect data from a larger range of $\mathbf{Q}$ values in the $b c$ plane. In Fig. 3 we compare a threedimensional color map of the most intense spin-wave dispersion measured at IN8 (a) to the results of our model calcu-
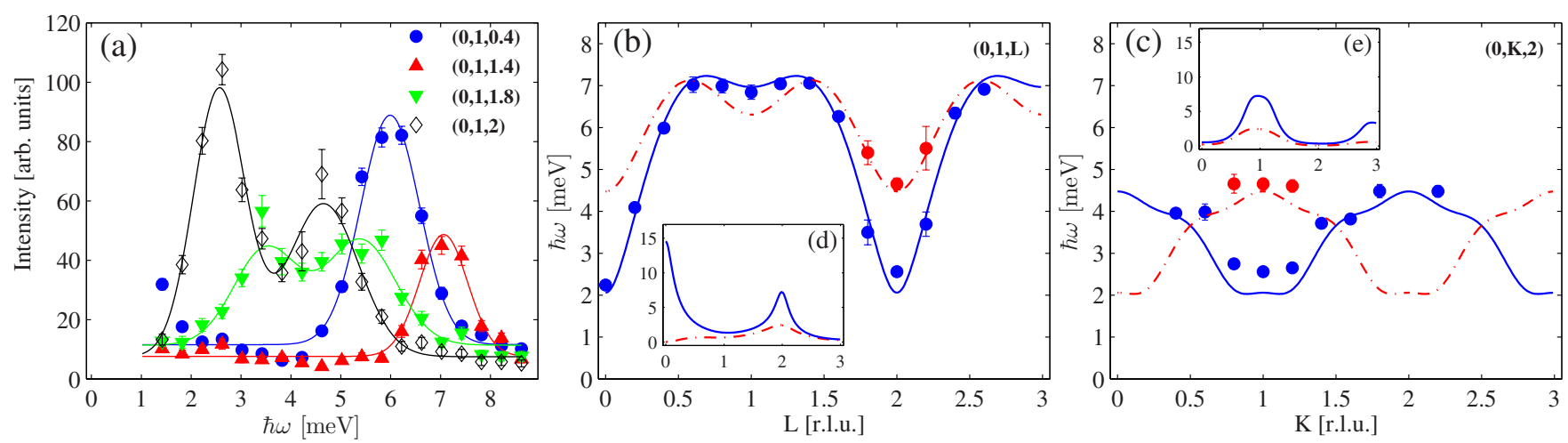

FIG. 4. (Color online) (a) Constant $\mathbf{Q}=(0,1, L)$ cuts from IN8. At $\mathbf{Q}=(0,1,0.4)$ and $(0,1,1.4)$ only a single branch is visible, but at $\mathbf{Q}=(0,1,1.8)$ and $(0,1,2)$ both branches are observed. (b) Dispersions along $(0,1, L)$ measured at IN8 [blue (black) and red (gray) circles compared to the calculated spin-wave energies (full and dotted lines). The inset shows the calculated intensity of the dispersions. Two branches are observed at $\mathbf{Q}$ where the model predicts spin-waves with finite intensity that are well separated in energy. (c) Dispersions along $(0, K, 2)$ measured at IN8 and compared to the calculated spin-wave energies. The inset shows the calculated intensity of the branches. Along $(0, K, 0)$ [Fig. 1(b)] the intensity of the second branch is always zero, but here, along $(0, K, 2)$, there are $\mathbf{Q}$-values where both branches have intensity and can be observed. 
lation (b) using the interaction parameters of Table I. The experiment at IN8 gave clear evidence of two nondegenerate magnon branches, as seen in Fig. 4. Here two-peak lineshapes were observed in energy scans at scattering vectors where the calculations predicted that the branches were well separated in energy and both had finite intensity.

The exchange interactions established in this work may be used to explain the magnetic ground state. Maximizing $J(\mathbf{Q})=\sum J_{i j} e^{-i \mathbf{Q} \cdot \mathbf{R}_{i j}}$ at zero temperature shows that the IC order is favorable in a simple model of layered magnetic systems with competing interactions if the effective exchange interactions, $J_{1}$ and $J_{2}>0$, between $\mathrm{NN}$ and NNN ferromagnetic layers fulfill that $\left|J_{1}\right|<4 J_{2} \cdot{ }^{18}$ If this condition applies, the magnetic ordering vector $Q$ is determined by $\cos (Q d)=$ $-J_{1} / 4 J_{2}$, where $d$ is the interlayer distance between adjacent ferromagnetic layers. Since $\mathrm{LiNiPO}_{4}$ has ferromagnetic layers perpendicular to the $b$ direction, we can test this condition. Here $J_{1}=2 J_{b c}+2 J_{a b}$ and $J_{2}=J_{b}$, while $d=b / 2$. Using the exchange parameters in Table I we find: $J_{1}=2.7(2) \mathrm{meV}$ and $4 J_{2}=2.68(4) \mathrm{meV}$, for the competing interactions. Within the uncertainties the magnetic ground state could therefore be either $\mathrm{C}$ or IC, and the system is close to an instability. A mean-field model using interaction parameters obtained from the spin-wave spectra predicts a behavior qualitatively similar to that observed experimentally. ${ }^{19}$ Here it is found that the magnetic structure is IC just below $T_{\text {IC }}$, but that the extra lock-in anisotropy energy gained by the $\mathrm{C}$ structure becomes important at slightly lower temperatures and as result changes $q$ from $\mathbf{k}_{\mathrm{IC}}$ to 0 .

Jens Jensen is greatly acknowledged for illuminating discussions. Work was supported by the Danish Natural Science Research Council under DANSCATT, and by the Swiss NSF Contracts No. PP002-102831 and No. 200020-105175. This research project is based on experiments performed at the Swiss spallation neutron source SINQ, Paul Scherrer Institute, Villigen, Switzerland, Institute Laue Langevin, and HFIR. This Brief Report has been authored, in whole or in part, under Contract No. DE-AC02-07CH11358 with the U.S. Department of Energy.
${ }^{1}$ S.-W. Cheong and M. Mostovoy, Nature Mater. 6, 13 (2007).

${ }^{2}$ M. Kenzelmann, A. B. Harris, S. Jonas, C. Broholm, J. Schefer, S. B. Kim, C. L. Zhang, S. W. Cheong, O. P. Vajk, and J. W. Lynn, Phys. Rev. Lett. 95, 087206 (2005).

${ }^{3}$ W. Eerenstein, N. D. Mathur, and J. F. Scott, Nature (London) 442, 759 (2006).

${ }^{4}$ M. Mercier, Ph.D. thesis, Université de Grenoble, 1969.

${ }^{5}$ J.-P. Rivera, Ferroelectrics 161, 275 (1993).

${ }^{6}$ B. B. van-Aken et al., Nature (London) 449, 702 (2007).

${ }^{7}$ T. B. S. Jensen et al., preceding paper, Phys. Rev. B 79, 092412 (2009).

${ }^{8}$ D. Vaknin, J. L. Zarestky, J. P. Rivera, and H. Schmid, Phys. Rev. Lett. 92, 207201 (2004).

${ }^{9}$ I. Abrahams and K. S. Easson, Acta Crystallogr., Sect. C: Cryst. Struct. Commun. 49, 925 (1993).

${ }^{10}$ C. R. H. Bahl et al., Nucl. Instrum. Methods Phys. Res. B 246, 452 (2006).

${ }^{11}$ F. Demmel, N. Grach, and H. M. Rønnow, Nucl. Instrum. Meth- ods Phys. Res. A 530, 404 (2004).

${ }^{12}$ R. P. Santoro, D. J. Segal, and R. E. Newnham, J. Phys. Chem. Solids 27, 1192 (1966); R. P. Santoro and R. E. Newnham, Acta Crystallogr. 22, 344 (1967).

${ }^{13}$ D. Vaknin, J. L. Zarestky, J. E. Ostenson, B. C. Chakoumakos, A. Goni, P. Pagliuso, T. Rojo, and G. E. Barberis, Phys. Rev. B 60, 1100 (1999).

${ }^{14}$ T. B. S. Jensen, Ph. D. thesis, Risø DTU and University of Copenhagen, 2007, www.bricksite.com/tstibius.

${ }^{15}$ J. L. Zarestky et al., J. Magn. Magn. Mater. 234, 401 (2001).

${ }^{16}$ P. A. Lindgård, A. Kowalska, and P. Laut, J. Phys. Chem. Solids 28, 1357 (1967).

${ }^{17}$ Since the energy gap does not change significantly between 2 and $10 \mathrm{~K}$ we used the same interaction parameters when fitting at 2 and $10 \mathrm{~K}$.

${ }^{18}$ T. Nagamiya, in Solid State Physics, edited by F. Seitz and D. Turnbull (Academic, New York, 1967), Vol. 29, p. 346.

${ }^{19} \mathrm{~J}$. Jensen (private communication). 